Neue Pflegeserie mit AntifaltenEffekt

Wer trockene oder sehr trockene Haut hat, erwartet von der idealen Hautcreme in erste Linie, dass sie Spannungsgefühle und Juckreiz lindert, Schuppigkeit reduziert und den Hautzustand stabilisiert. Das Produkt sollte natürlich auch Falten mildern können. Um allen Anforderungen gerecht zu werden, hat Eucerin nun die erste Spezialpflege für trockene bis sehr trockene Haut mit Antifalteneffekt entwickelt. Sie vereint die beiden bewährten Wirkstoffe Urea und Hyaluronsäure in einer Formel. Die Pflegeserie umfasst drei Produkte: der Eucerin ${ }^{\circledR}$ Hyal-Urea Antifalten Tagescreme, Eucerin ${ }^{\circledR}$ Hyal-Urea Antifalten Nachtcreme und Eucerin ${ }^{\circledR}$ Hyal-Urea Antifalten Augencreme.

Nach Informationen von Beiersdorf

\section{Mehr vom Sonnenschutz}

Das liposomale Sonnenschutzmittel Daylong ${ }^{\circledR}$ Kids ist jetzt auch in der neuen Flaschengröße von $200 \mathrm{ml}$ erhältlich. Die größere Flasche bietet gegenüber der bisherigen 100-mlFlasche einen klaren Preisvorteil: Sie ist für 24,95 Euro exklusiv in der Apotheke erhältlich. Daylong ${ }^{\circledR}$ Kids wurde speziell für die sensible Kinderhaut entwickelt. Neben dem hohen LSF von 30 ist es schweißfest, wasserabweisend und enthält weder Duftstoffe noch PEGEmulgatoren.

Nach Informationen von Spirig Pharma

\section{Wundauflage in neuen Größen}

Urogtül ${ }^{\circledR}$ Soft, eine TLC-Wundauflage, die sich aufgrund ihrer Flexibilität und besonders guten Modellierbarkeit auch für schwierige anantomische Verhältnisse sehr gut eignet, ist jetzt noch flexibler anwendbar. Seit Juli steht die vielseitig einsetzbare Wundauflage in den neuen Abmessungen $5 \times 5 \mathrm{~cm}$, $10 \times 40 \mathrm{~cm}$ und $20 \times 30 \mathrm{~cm}$ zur Verfügung. Mit diesem variablen Angebot kann man auf die unterschiedlichsten Wundsituationen reagieren. Urogotül ${ }^{\circledR}$ Soft in der Abmessung $5 \times 5 \mathrm{~cm}$ ist zudem jetzt in der praxigerechten Spenderbox à 120 Stück erhältlich.

Nach Informationen von Urgo Medical

\title{
Aktinische Keratosen ernst nehmen
}

— Die aktinische Keratose (AK) hat in den vergangenen Jahren einen deutlichen Wandel erlebt. Sie wird heute als Schlüssel zum Plattenepithelkarzinom (PEC) angesehen. Entsprechend erscheint sie in einer Leitlinie der DDG von 2004 als PEC der Epidermis in situ, berichtete Dr. Claas Ulrich, Berlin. Damit haben sich auch die Ziele in der Therapie der AK über die Jahre geändert. Ging es früher vorrangig um kosmetische Belange und die Prävention von Rezidiven, steht heute die Prävention invasiver PEC im Vordergrund.

Zu den zugelassenen Flächentherapien der AK, die so früh wie möglich zum Einsatz kommen sollten, gehört die pro-apoptotisch wirkende Kombination aus 3\% Diclofenac in 2,5\% Hyaluronsäure als Trägersubstanz (Solaraze ${ }^{\circledR}$ Gel). Die Wirkung dieses Gels basiert auf der Cyclooxygenase-2(COX-2)Expression in der AK. Über die Hemmung von COX-2 wird die Prostaglandin(PGE2)Synthese in Tumorzellen inhibiert und Signalwege getriggert, die die Apoptose fördern. Dass dieses Prinzip nachhaltig klinisch wirksam ist, belegt eine offene placebo- kontrollierte Phase-IV-Studie aus den USA. Die Therapie der AK-Patienten mit zweimal täglich Solaraze ${ }^{\circledR}$-Gel dauerte 90 Tage. In der Nachbeobachtungsphase hatten 91\% der Patienten nach einem Jahr bezogen auf die anfänglich vorhandenen Läsionen eine $75 \%$ ige und $70 \%$ eine $100 \%$ ige Clearance erreicht. Die entsprechenden Erfolgsquoten bei den anfänglich identifizierten plus den neu hinzugekommen Läsionen lagen bei 79 und $30 \%$.

Ulrich et al. untersuchten in einer Studie bei organtransplantierten (Niere, Herz, Leber) Patienten mit AK Verträglichkeit und Wirksamkeit des Diclofenac/Hyaluronsäure-Gels über eine 16 Wochen dauernde Behandlung. Sie erreichten damit im Mittel eine komplette Clearancerate von etwa $45 \%$.

mb

Mittagsseminar „Behandlung epithelialer Tumoren - State of the Art", anlässlich der 22. Fortbildungswoche für praktische Dermatologie und Venerologie, München, 28.7.2010 (Veranstalter: Almirall Hermal)

\section{Proaktiv gegen Ekzemrezidive}

— Häufige Rezidive erschweren das Langzeit-Management des atopischen Ekzems (AE). Proaktive Therapie mit Advantan ${ }^{\circledR}$, einem topischen Glukokortikoid der 4. Generation, trägt dazu bei, die Krankheitsaktivität auch langzeitig zu kontrollieren

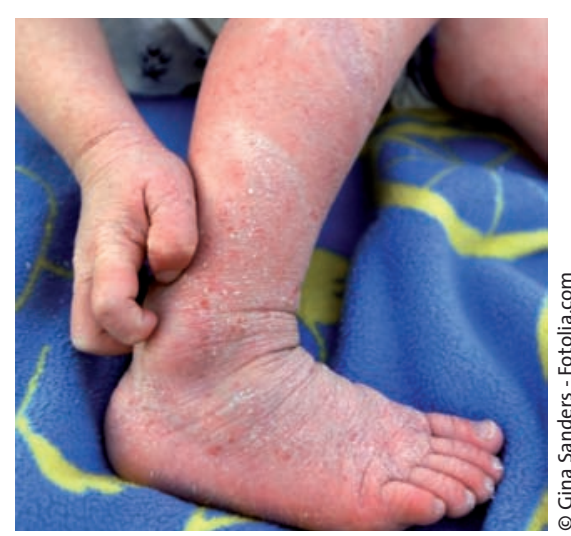

Atopisches Ekzem: Geeignete Prävention senkt die Rezidivrate.
- dies ist inzwischen auch durch Studienergebnisse belegt.

Bei der proaktiven Therapie folgt auf eine kurzzeitige Akuttherapie mit täglicher Applikation eines topischen Kortikoids eine längerfristige Erhaltungstherapie, beispielsweise mit zweimal wöchentlicher Anwendung des Glukokortikoids, kombiniert mit Basistherapie. Unter diesem Ansatz fiel in Studien die Rezidivrate 3,5-mal geringer aus als unter alleiniger Basistherapie.

Auch nach 16-wöchiger Anwendung bei zweimal wöchentlichen Anwendung von Advantan ${ }^{\circledR}$ zeigten sich keine steroidinduzierten Nebenwirkungen. Die Substanz ist in fünf verschiedenen Formulierungen erhältlich: Creme, Salbe, Lösung, Fettsalbe oder Milch - und damit für jeden Hautzustand und jedes Lebensalter verfügbar. red

Nach Informationen von Intendis 